\title{
DURAÇÃO DO PERÍODO NINFAL E SOBREVIVÊNCIA DO PREDADOR PODISUS CONNEXIVUS BERGROTH (HEMIPTERA, PENTATOMIDAE), EM TRÊS PRESAS ALTERNATIVAS ${ }^{1}$
}

\author{
José Cola Zanuncio ${ }^{2}$ \\ Joaquim Efigênio M. Leite ${ }^{3}$ \\ João Batista Alves ${ }^{4}$ \\ Germi Porto Santos 5
}

\begin{abstract}
NINFAL PERIOD DURATION AND SURVIVAL OF THE PREDATOR PODISUS CONNEXIVUS BERGROTH (HEMIPTERA, PENTATOMIDAE), IN THREE ALTERNATIVE PREYS. Longevity and survival of the predator Podisus connexivus (Hemiptera: Pentatomidae) were studied in three alternative preys: $\mathrm{T}_{1}-$ Bombyx mori (Lepidoptera, Bombycidae) catterpilars; $\mathrm{T}_{2}$ - Musca domestica (Diptera, Muscidae) larva and $\mathrm{T}_{3}-$ Tenebrio molitor (Coleoptera, Tenebrionidae) larva. Longevity and survival were: $22,1 \pm 0,6$ days and $54,3 \pm 5,3 \% ; 25,2 \pm 1,3$ days and $56,0 \pm 4,9 \%$ and $22,0 \pm 0,8$ days and $34,6 \pm 8,6 \%$, for treatments $\mathrm{T}_{1}, \mathrm{~T}_{2}$ and $\mathrm{T}_{3}$, respectively. Comparing to other researches, a lower survival was found. This is probably because a $F_{2}$ generation from field material, was used. Since the insect was not well adapted to the laboratory conditions this could have led to lower survival.

KEY WORDS. Hemiptera, Pentatomidae, Podisus connexivus, ecology
\end{abstract}

A expansão da fronteira agropecuária, para atender a demanda da população nos seus requisitos básicos, tem gerado uma série de problemas e entre os quais, se destaca a ação das pragas sobre as diversas culturas. No setor florestal, a situação é semelhante e, atualmente no Brasil, um dos maiores problemas consiste no controle de pragas, notadamente das lagartas desfolhadoras, sem se lançar mão dos produtos químicos.

Para o ecossistema florestal, o manejo integrado de pragas é, sem sombra de dúvidas, o caminho a ser buscado e, neste contexto, a ação do controle biológico é de importância fundamental, com a finalidade de se manter as pragas em um nível de dano suportável pela cultura e que não proporcione danos econômicos.

1) Pesquisa apoiada pelo BIAGRO, CNPq, FAPEMIG, CAF Florestal e REFLORALJE/SIF.

2) Universidade Federal de Viçosa, 36570-000 Viçosa, Minas Gerais, Brasil. Bolsista do CNPq.

3) Curso de Pós-graduação em Entomologia, Universidade Federal de Viçosa, 36570-000 Viçosa, Minas Gerais, Brasil.

4) CODEMIN S.A., Caixa Postal 36, 77160-970 Niquelândia, Goiás, Brasil.

5) Empresa Brasileira de Pesquisa Agropecuária, Caixa Postal 216, 36570-000 Viçosa, Minas Gerais, Brasil. Bolsista do CNPq. 
A utilização do controle biológico em todo o mundo, é uma prática antiga. Inicialmente de maneira empírica e, com o passar dos anos, aperfeiçoada à medida em que se desenvolveram técnicas de criação massal de inimigos naturais. Dentre os agentes de controle biológico, a atuação de predadores é importante, pois de acordo com DEBACH (1964), metade das espécies da classe Insecta é constituída por parasitóides e predadores, sendo mais freqüente a predação que o parasitismo de insetos por insetos.

De acordo com CLAUSEN (1940) e HAGEN et al. (1976), a ordem Hemiptera, ocupa o quarto lugar na classe Insecta, apresenta 22 famílias com espécies predadoras, durante todo ou apenas em uma fase do seu ciclo biológico. SILVA (1933), BUCKUP (1960) e BUCKUP (1961) mencionam que, além das espécies fitófagas, a família Pentatomidae apresenta na sub-família Asopinae, 32 espécies predadoras, encontrando-se neste grupo, o gênero Podisus.

No Brasil, vários trabalhos foram executados, procurando-se desenvolver técnicas de criação para esse importante grupo de predadores, conforme BUENO (1982), com a biologia de Montina confusa; GONÇALVES (1990), com a biologia de Podisus nigrolimbatus e Podisus connexivus; ZANUNCIO et al. (1990), com a biologia de P. connexivus, alimentado em lagartas de Bombyx mori (Lepidoptera, Bombycidae) e larvas de Musca domestica (Diptera, Muscidae) e BARCELOS (1991), com a biologia de $P$. nigrolimbatus sobre lagartas de B. mori.

O presente trabalho teve por objetivo, estudar a duração e sobrevivência do período ninfal de $P$. connexivus, alimentado em três presas alternativas e, desta forma, propiciar informações que possam auxiliar em pesquisas futuras à respeito desse importante predador.

\section{MATERIAL E MÉTODOS}

Esta pesquisa foi desenvolvida com ninfas de terceira geração, de $P$. connexivus, no Laboratório de Entomologia Florestal da Universidade Federal de Viçosa, sob condições controladas de temperatura, umidade e fotoperíodo de $25 \pm 2^{\circ} \mathrm{C}, 70 \pm 10 \%$ e 12 horas, respectivamente. Os ovos, foram obtidos de várias fêmeas, a fim de obter-se maior variabilidade genética, e acondicionados em placas de Petri de $9 \mathrm{~cm}$ de diâmetro por $1,5 \mathrm{~cm}$ de altura. Junto a esses, colocou-se um chumaço de algodão embebido em água destilada. O delineamento experimental constituiu-se de parcelas com 10 ninfas, inteiramente casualizadas, com três tratamentos e 15 repetições. Os tratamentos foram: ninfas alimentadas em lagartas de B. mori. $\left(\mathrm{T}_{1}\right)$, ninfas alimentadas em larvas de Musca domestica (Diptera, Muscidae) $\left(\mathrm{T}_{2}\right)$ e ninfas alimentadas em larvas de Tenebrio molitor (Coleoptera, Tenebrionidae) $\left(\mathrm{T}_{3}\right)$. Cada parcela foi constituída por 10 ninfas de segundo ínstar, acondicionadas em potes plásticos de $500 \mathrm{ml}$, com tampa plástica, na qual foi feito um orifício de quatro centímetros de diâmetro no centro. Neste local foi inserido um copo plástico de $40 \mathrm{ml}$ (tipo cafezinho), com o fundo constituído por tela de nailon, no qual colocavam-se as 
presas, para a alimentação das ninfas. Todos os dias, era feita a remoção das presas mortas e ou sugadas, substituindo-as por outros vivas. A água era fornecida por um tubo de plástico ou vidro tipo anestésico odontológico, inserido na tampa do recipiente de criação. Para evitar o escoamento da água e facilitar a sua utilização pelas ninfas, colocava-se um chumaço de algodão na extremidade inferior do tubo. Os resultados obtidos nos três tratamentos foram analisados estatisticamente através da análise de variância e as médias comparadas pelo teste de Tukey ao nível de $5 \%$ de probabilidade.

\section{RESULTADOS E DISCUSSÃO}

O comportamento e as diferenças de longevidade e sobrevivência da fase ninfal são apresentados nos três tratamentos (Tab. I).

Tabela I. Longevidade (dias) de cada ínstar do predador Podisus connexivus Bergroth, 1891 (Hemiptera, Pentatomidae), para os tratamentos: $\mathrm{T}_{1}=$ lagartas de Bombyx mori; $\mathrm{T}_{2}=$ larvas de Musca domestica e $T_{3}=$ larvas de Tenebrio molitor. Viçosa, Minas Gerais, 1990. I, II, III, IV e $\mathrm{V}=$ ínstares; $\mathrm{D} . \mathrm{F} .=$ duração da fase; $\mathrm{X}=$ média; $\mathrm{A} . \mathrm{V}$. = amplitude de variação.

\begin{tabular}{|c|c|c|c|c|c|c|c|c|c|c|c|c|}
\hline & \multicolumn{2}{|c|}{ I } & \multicolumn{2}{|c|}{ II } & \multicolumn{2}{|c|}{ III } & \multicolumn{2}{|c|}{ IV } & \multicolumn{2}{|c|}{ v } & \multicolumn{2}{|c|}{ D.F. } \\
\hline & $\mathrm{x}$ & A.V. & $x$ & A.V. & $\bar{x}$ & A.V. & $\bar{x}$ & A.V. & $\vec{x}$ & A.V. & $\bar{x}$ & A.V. \\
\hline$T_{i}$ & 4,0 & 0,0 & $4,3 \pm 0,3 a$ & $3,0-5,4$ & $3.9+0.2 \mathrm{a}$ & $2,8-5,9$ & $3,8 \pm 0,2 \mathrm{a}$ & $2,8-6,3$ & $6,0+0,5 \mathrm{a}$ & $3,8-10,2$ & $22,1 \pm 0,6 \mathrm{a}$ & $19,4-28,1$ \\
\hline $\mathbf{T}_{2}$ & 4,0 & 0,0 & $4,2+0,1 \mathrm{a}$ & $3,6-5,2$ & $3,6 \pm 0,3 \mathrm{a}$ & $2,1-7.4$ & $5,0 \pm 0,5 \mathrm{~b}$ & $2,6-8,3$ & $8,0 \div 0,8 \mathrm{~b}$ & $4,7-16,5$ & $25,2+1,3 \mathrm{~b}$ & $18,9-37,2$ \\
\hline $\mathrm{T}_{3}$ & 4,0 & 0,0 & $4,3+0,3 \mathrm{a}$ & $3,4 \cdot 7,4$ & $4,0 \pm 0,2 \mathrm{a}$ & $3,1-5,0$ & $3,9 \pm 0,4 a$ & $1,0-8,0$ & $5,5 \pm 0,5 \mathrm{a}$ & $3,0-9,0$ & $22,0 \pm 0,8 \mathrm{a}$ & $19,8-24,3$ \\
\hline
\end{tabular}

* Médias seguidas da mesma letra não diferem entre si pelo Teste de Tukey ao nível de $5 \%$ de probabilidade.

\section{LONGEVIDADE}

Primeiro ínstar. As ninfas apresentam comportamento bastante peculiar, ou seja, permanecem agrupadas e após sugarem os restos dos fluídos dos ovos, deslocam-se para o algodão embebido em água. A duração neste ínstar foi de quatro dias e não há necessidade de alimentação suplementar.

Segundo ínstar. As ninfas perdem o hábito gregário e locomovem-se com bastante rapidez, à procura de presas, iniciando-se assim, a alimentação diferenciada. As ninfas se agrupam somente para atacar uma presa com tamanho superior a elas, o que diminue o tempo para paralisar e matar a mesma. Este comportamento foi comum durante este ínstar, na alimentação com lagartas de B. mori e larvas de T. molitor. No entanto, com larvas de $M$. domestica, somente no primeiro e segundo dia de alimentação, observou-se várias ninfas alimentando-se em um mesmo indivíduo. À medida que essas se desenvolviam, era comum o ataque de, no máximo três indivíduos em cada larva de $M$. domestica. Quanto à duração deste ínstar, não houve diferença significativa entre os tratamentos $T_{1}, T_{2}$ e $T_{3}$, que foram, respectivamente, de 4,3 $\pm 0,3$; $4,2 \pm 0,1$ e 4,3 $\pm 0,3$ dias. 
Terceiro ínstar. As ninfas apresentam comportamento semelhante ao ínstar anterior, com movimentação um pouco mais moderada, sendo pouco comum se observar mais de uma delas predando a mesma larva de $M$. domestica (talvez pelo seu pequeno porte). No entanto, nas outras presas, o hábito de alimentação em grupo continuou até a fase adulta. Também, à exemplo da fase anterior, a longevidade entre os três tratamentos não apresentou diferença significativa e foi de $3,9 \pm 0,2 ; 3,6 \pm 0,3$ e $4,0 \pm 0,2$ dias para $T_{1}, T_{2}$ e $T_{3}$, respectivamente. Os resultados indicam claramente uma tendência de redução na duração do período ninfal até o terceiro ínstar, para as ninfas que receberam larvas de $M$. domestica, ou seja, um tempo acumulado de 11,8 dias contra 12,2 e 12,3 dias para os tratamentos com lagartas de B. mori e larvas de T. molitor, respectivamente.

Quarto ínstar. As ninfas se locomovem mais que o estágio anterior, são mais ágeis e agressivas, chegando muitas vezes a saltar de um lugar mais alto para outro mais baixo. Com relação à longevidade deste ínstar, os valores apresentados para os tratamentos $\mathrm{T}_{1}, \mathrm{~T}_{2}$ e $\mathrm{T}_{3}$, foram respectivamente de $3,8 \pm$ 0,$2 ; 5,0 \pm 0,5$ e $3,9 \pm 0,4$ dias. Pelo teste de média, houve diferença significativa ao nível de $5 \%$ de $\mathrm{T}_{2}$ para $\mathrm{T}_{1}$ e $\mathrm{T}_{3}$ que por sua vez, mostraram-se estatisticamente iguais. A partir deste ínstar, as ninfas alimentadas com lagartas de B. mori passaram a apresentar uma tendência de menor tempo acumulado de duração que para os outros tratamentos, ou seja, de 16 dias contra 16,8 e 16,2 para aquelas que receberam larvas de $M$. domestica e de $T$. molitor, respectivamente.

Quinto ínstar. As ninfas reduzem seus movimentos, locomovem-se muito pouco e apresentam agressividade mais moderada. A longevidade foi de $6,0 \pm 0,5 ; 8,0 \pm 0,8$ e $5,5 \pm 0,5$ dias para $T_{1}, T_{2}$ e $T_{3}$, respectivamente. Ninfas alimentadas com larvas de $M$. domestica apresentaram maior agressividade e este resultado foi significativamente superior aos outros dois tratamentos, que por sua vez foram iguais entre si.

Considerando-se a duração total da fase ninfal, verifica-se que as melhores performances foram obtidas com ninfas criadas em lagartas de $B$. mori $\mathrm{e}$ larvas de $T$. molitor, com valores semelhantes entre si, $22,1 \pm 0,6$ e 22,0 00,8 dias, respectivamente e, que por sua vez foram diferentes do apresentado por ninfas criadas com larvas de $M$. domestica, ou seja uma duração de $25,2 \pm 1,3$ dias.

\section{SOBREVIVÊNCIA}

No primeiro ínstar, quando não havia ainda a influência dos tratamentos, a sobrevivência das ninfas foi de $100 \%$. A partir do segundo ínstar, a sobrevivência foi de $86,6 \pm 4,5 ; 91,3 \pm 2,4$ e $72,6 \pm 9,4 \%$ para $T_{1}, T_{2}$ e $T_{3}$, respectivamente, não apresentando diferenças significativas entre as mesmas. No terceiro ínstar a sobrevivência foi de $97,2 \pm 1,7 ; 86,1 \pm 3,8$ e $99,2 \pm 0,8 \%$, ocorrendo diferença significativa somente entre o tratamento $T_{2}$, enquanto os outros dois foram iguais entre si. No quarto ínstar, o tratamento $\mathrm{T}_{3}$ diferiu dos outros dois, com valor de $91,4 \pm 4,3$ contra $87,7 \pm 2,2$ e $83,7 \pm 4,3 \%$ para $T_{1}$ e $T_{2}$, respectivamente. No quinto e último ínstar, a sobrevivência foi estatisticamente igual para os 
tratamentos $\mathrm{T}_{1}$ e $\mathrm{T}_{2}$ com valores de $67,6 \pm 6,6$ e 79,9 $44,5 \%$, sendo superiores ao tratamento $\mathrm{T}_{3}$ que apresentou $54,9 \pm 0,4 \%$ (Tab. II). Verifica-se que não houve uma constância de vantagem, deste ou daquele tratamento, ao longo desta fase, ocorrendo inversões de valores, dependendo do estágio ninfal e do tipo de tratamento. Todavia, quando se analisa este parâmetro ao final do ciclo, constata-se que o pior desempenho foi atribuído às ninfas alimentadas em larvas de T. molitor, com uma média de sobrevivência de $34,6 \pm 8,6 \%$ contra $54,3 \pm 5,3$ e $56,0 \pm 4,9 \%$ para os outros dois tratamentos, que foram iguais entre si.

Tabela II. Porcentagem de sobrevivência de cada ínstar do predador Podisus connexivus Bergroth, 1891 (Hemiptera, Pentatomidae), segundo os tratamentos: $\mathrm{T}_{1}=$ lagartas Bombyx mori; $\mathrm{T}_{2}=$ larvas de Musca domestica e T $3=$ larvas de Tenebrio molitor. Viçosa, Minas Gerais, 1990. I, II, III, IV e $\mathrm{V}=$ ínstares; \% S.C.T. $=$ porcentagem de sobrevivência no ciclo total; $\mathrm{X}=$ média; $\mathrm{A} . \mathrm{V} .=$ amplitude de variação.

\begin{tabular}{|c|c|c|c|c|c|c|c|c|c|c|c|c|}
\hline & \multicolumn{2}{|c|}{ I } & \multicolumn{2}{|c|}{ II } & \multicolumn{2}{|c|}{ III } & \multicolumn{2}{|c|}{ IV } & \multicolumn{2}{|c|}{ V } & \multicolumn{2}{|c|}{ \% S. C. T. } \\
\hline & $\bar{x}$ & A.V. & $\bar{x}$ & A.V. & $\bar{x}$ & A.V. & $\bar{X}$ & A.V. & $\bar{X}$ & A.V. & $\bar{x}$ & A.V. \\
\hline$T_{1}$ & 100 & 0,0 & $86,6+4,5 a$ & $50,0-100$ & $97,2 \pm 1,7 \mathrm{a}$ & $77,7-100$ & $87,7 \pm 2,2 \mathrm{a}$ & $77,7-100$ & $67,6 \pm 6,6 a$ & $0,0-100$ & $54,3 \pm 5,3 \mathrm{a}$ & $0,0-100$ \\
\hline $\mathrm{T}_{2}$ & 100 & 0,0 & $91,3 \pm 2,4 \mathrm{a}$ & $70,0-100$ & $86,1+3,8 a$ & $77,7-100$ & $83,7 \pm 4,3 a$ & $35,5-100$ & $79,9 \pm 4,5 a$ & $60,0-100$ & $56,0 \pm 4,9 a$ & $20,0-80$ \\
\hline $\mathrm{T}_{3}$ & 100 & 0,0 & $72,6 \pm 9,4 a$ & $0,0-100$ & $99,2 \pm 0,8 \mathrm{a}$ & $90,0-100$ & $91,4 \pm 4,3 b$ & $50,0-100$ & $54,9 \pm 0,4 \mathrm{~b}$ & $0,0-100$ & $34,6 \pm 8,6 \mathrm{~b}$ & $0,0-90$ \\
\hline
\end{tabular}

* Médias seguidas da mesma letra não diferem entre si pelo Teste de Tukey ao nível de 5\% de probabilidade.

Considerando-se os aspectos de mão-de-obra, aquisição da presa, custos e outros fatores, as poucas diferenças biológicas no desenvolvimento de $P$. connexivus alimentado em lagartas de $B$. mori e larvas de $M$. domestica, demonstra que esta última presa é bastante promissora e supre muito bem as exigências nutricionais do predador. Embora a presa alternativa que mais se aproxima das naturais seja $B$. mori, pôde-se demonstrar nesse trabalho que é possível a produção de $P$. connexivus, com vantagem, em $M$. domestica, que é uma presa de mais fácil criação. Assim, esse inseto pode substituir a presa comumente utilizada, ou seja, lagartas de B. mori.

AGRADECIMENTOS. Ao BIOAGRO, CNPq, FAPEMIG pelas bolsas e auxílios concedidos. A CAF Florestal Ltda, REFLORALJE S.A. e Sociedade de Investigações Florestais (SIF),pelo apoio na realização deste trabalho.

\section{REFERÊNCIAS BIBLIOGRÁFICAS}

BARCELOS, J.A.V. 1991. Biologia do predador Podisus nigrolimbatus (Hemiptera: Pentatomidae) sobre lagartas vivas ou previamente congeladas de Bombyx mori (Lepidoptera: Bombycidae) em laboratório. Tese de Mestradọ, não publicada, Universidade Federal de Viçosa, Viçosa, 86p.

BUCKUP, L. 1960. Contribuição para o conhecimento de Asopinae (Hemiptera: Pentatomidae) da América do Sul. Iheringia, Série Didática, 15: 1-25. 


\section{Série Didática, 6: 1-24.}

BUENO, V.H.P. Biologia e aspectos morfológicos de Montina confusa (Stal, 1859) (Hemiptera: Reduviidae, Zelinae). Tese de Mestrado, não publicada, Escola Superior de Agricultura Luiz de Queiroz, Piracicaba, 90p.

CLAUSEN, C.P. 1940. Entomophagous Insects. New York, McGraw-Hill, $688 p$.

DEBACH, P.1964. Biological Control of Insect Pests and Weeds. London, Chapmam \& Hall, 844p.

GONÇALVES, L. 1990. Biologia e capacidade predatória de Podisus nigrolimbatus Spínola, 1835 e Podisus connexivus Bergroth, 1891 (Hemiptera: Pentatomidae, Asopinae) em condições de laboratório. Tese de Mestrado, não publicada, Escola Superior de Agricultura Luiz de Queiroz, Lavras, 87p.

HAGEN, K.S.; S. BOMBOCHI \& J.A. MCMURTHY. 1976. The biology and impact of predators. In: C.B. HUFFAKER \& P.S. MESSENGER (ed.). Theory and Pratics of Biological Control. San Francisco Academic Press, $788 \mathrm{p}$.

SILVA, A.G.A. 1933. Contribuição para o estudo da biologia de três pentatomídoes e o possível emprego destes insetos no combate biológico de espécies nocivas. O Campo 43 (3): 23-25.

ZANUNCIO, J.C.; J.B. ALVES; J.E.M. LEITE \& N.R. SILVA. 1990. Desenvolvimento ninfal do predador Podisus connexivus Bergroth, 1891 (Hemiptera: Pentatomidae) alimentados em diferentes proporções de lagartas de Bombyx mori e larvas de Musca domestica. Revista Árvore 14 (2): 164-174.

Recebido em 29.VII.1991; aceito em 25.XI.1993. 